\title{
Unveiling the Local Structure of Palladium Loaded into Imine-Linked Layered Covalent Organic Frameworks for Cross-Coupling Catalysis
}

\author{
Ignacio Romero-Muñiz,||Andreas Mavrandonakis,]Pablo Albacete, Alicia Vega, Valérie Briois, \\ Félix Zamora" und Ana E. Platero-Prats
}

\begin{abstract}
Layered covalent organic frameworks (2D-COFs), composed of reversible imine linkages and accessible pores, offer versatility for chemical modifications towards the development of catalytic materials. Nitrogen-enriched COFs are good candidates for binding Pd species. Understanding the local structure of reacting $P d$ sites bonded to the COF pores is key to rationalize interactions between active sites and porous surfaces. By combining advanced synchrotron characterization methods with periodic computational DFT modeling, the precise atomic structure of catalytic Pd sites attached to local defects is resolved within an archetypical imine-linked $2 D$ COF. This material was synthesized using an in situ method as a gel, under which imine hydrolysis and metalation reactions are coupled. Local defects formed in situ within imine-linked $2 D-C O F$ materials are highly reactive towards $P d$ metalation, resulting in active materials for Suzuki-Miyaura cross-coupling reactions.
\end{abstract}

\section{Introduction}

Covalent organic frameworks (COF) have been placed firmly in chemistry as unique porous architectures $\mathbf{M Q}$ for their use in applications related with water treatment, ${ }^{[\mathbf{B}}$ s sensing,

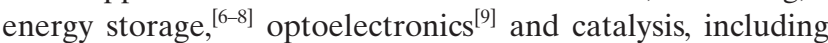

[*] I. Romero-Muñiz, P. Albacete, A. Vega, Prof. F. Zamora,

Dr. A. E. Platero-Prats

Departamento de Química Inorgánica

Facultad de Ciencias, Universidad Autónoma de Madrid

Campus de Cantoblanco, 28049 Madrid (Spain)

E-Mail: felix.zamora@uam.es ana.platero@uam.es

Dr. A. Mavrandonakis

Electrochemical Processes Unit, IMDEA Energy

Avenida Ramón de la Sagra 3, 28935 Móstoles, Madrid (Spain)

Dr. V. Briois

Synchrotron SOLEIL

L'Orme des Merisiers, Saint Aubin, BP48

91192 Gif-sur-Yvette Cedex (France)

Prof. F. Zamora, Dr. A. E. Platero-Prats

Condensed Matter Physics Center (IFIMAC)

Universidad Autónoma de Madrid

Campus de Cantoblanco, 28049 Madrid (Spain)

Prof. F. Zamora

Instituto de Investigación Avanzada en Ciencias Químicas de la UAM, Universidad Autónoma de Madrid

Campus de Cantoblanco, 28049 Madrid (Spain)

0 Sup

Supporting information and the ORCID identification number(s) for the author(s) of this article can be found under:

https://doi.org/10.1002/anie.202004197 photo- $[$ and electro-activated $\square$ reactions. A large family of these architectures are composed of designable organic building units linked through reversible covalent bonding, making COFs extremely light and flexible materials. COF synthesis is remarkably dynamic, evolving from highly disordered polymers at initial reaction times to porous crystalline frameworks at the end of the synthesis. The reversible nature of the COF linkages allows the starting disordered structures to reorganize into crystalline stable forms. Is indeed the intrinsic flexibility of these materials maybe one of their most unique yet underexplored structural features.

Among the expanding chemical variety of COF materials, imine-linked frameworks have been one of the leading players during the last years. from condensation of amine and aldehyde linkers often result

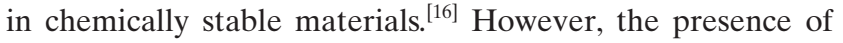
water can potentially reverse the imine formation, affording massive hydrolysis and collapse of the framework. An elegant strategy to avoid this undesired process has been reported by Banerjee and co-workers, based on replacing hydrolysable imine bonding by robust $\beta$-ketoenamine linkages, taking advantage of the irreversible imine-enamine tautomerism. 무 Simply by replacing a target trialdehyde building unit by its $\alpha$-substituted hydroxy analogue, a related $\beta$ ketoenamine-linked COF material with enhanced stability can be obtained. Recently, Dichtel and co-workers have reported a versatile synthetic strategy to prepare $\beta$-ketoenamine COF materials starting from their imine analogues through ligand exchange methods. ${ }^{\mathbb{Q} \mid}$ Interestingly, this subtle chemical modification of the COF architecture (that is, replacing imine by $\beta$-ketoenamine linkages) might result in structural variations of the framework, thereby playing a key role in the ultimate properties of the material. ${ }^{[\mathbf{Q}}$

Nitrogen-enriched COF materials are ideal candidates for binding catalytic metals, from single sites to few-atom clusters and bigger nanoparticles. carbon cross-couplings are unique tools in organic synthesis, in particular the widely used Suzuki-Miyaura reaction owing to the low toxicity of the coupling partners and the mild conditions required. ${ }^{\text {[4] }}$ One of the main challenges linked to this type of catalysis is, however, the recyclability of the palladium sites. One strategy is the use of structurally dynamic layered 2D-COF materials, where flexibility arises from the tunable arrangement of purely organic layers. As a proof of concept, Wang et al. in 2011 reported a pioneer work in which the synthesis of a 2D imine-COF loaded with $\mathrm{Pd}^{\mathrm{II}}$ pre-catalytic complexes that convert into $\mathrm{Pd}^{0}$ nano- 
particles during the Suzuki-Miyaura reactions is described. The direct incorporation of homogenous palladium nanoparticles into chemically modified 2D COF materials (that is, bearing ketoenamine or triazine groups) also resulted in good catalytic materials for one-pot Heck Sonogashira couplings [0] and multifold Heck reactions. [2] Also, palladium(II) complexes incorporated into an imine-linked triazine 2D-COF have been proved to catalyze Hiyama couplings. a 3D imine-linked COF material containing palladium acetate as heterogeneous catalyst showing excellent conversion rates in cross-coupling reactions under flow conditions was reported. ${ }^{\mathbb{9}}$ However, understanding the local nature of palladium species loaded into COF frameworks under conditions relevant for cross-coupling catalysis still remains under debate.

In the present work, we combine experimental probes of local and long-range atomic structure with computational modeling to provide a more realistic picture of the catalytic palladium species loaded into an archetypical defective imine-linked 2D COF material. In particular, we report a room-temperature one-pot method to bind palladium complexes into local defects within imine-linked 2D-COF platforms (Figure 1), affording active catalytic materials for Suzuki-Miyaura cross-coupling reactions. X-ray synchrotron pair distribution function (PDF) analyses and computational characterization indicate that palladium sites are thermodynamically stabilized when binding local defects containing amine terminations within the COF structures. Detailed analyses combining PDF, extended X-ray absorption fine structure (EXAFS) spectroscopy and density functional theory (DFT) modeling are applied to study in detail the real structural nature of the reacting palladium species within an archetypical defective imine-linked 2D-COF, under conditions relevant for palladium metalation and SuzukiMiyaura cross-coupling catalysis.

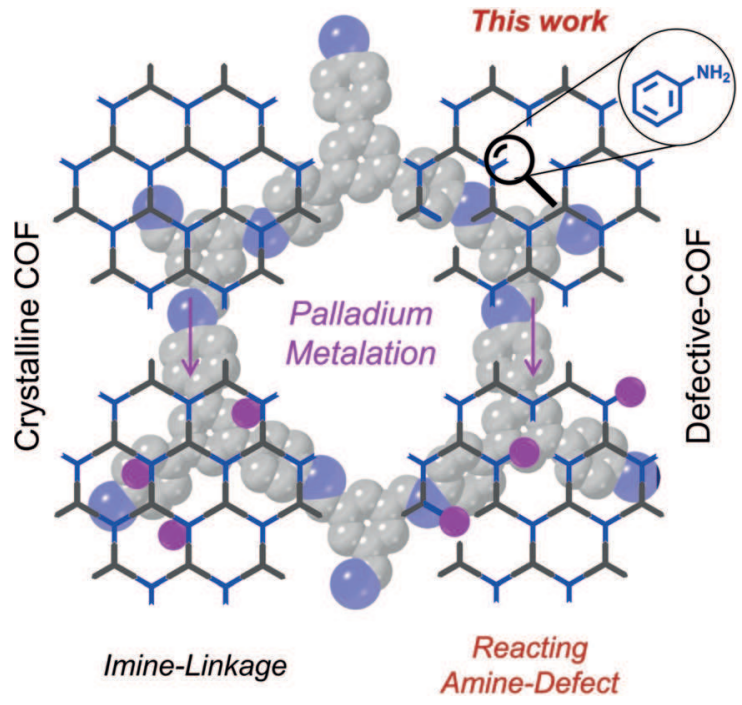

Figure 1. Left: Traditional metalation on a crystalline imine-linked COF material. Right: The in situ method developed herein for which defect healing and palladium binding are coupled.

\section{Results and Discussion}

The honeycomb imine-linked 2D-framework TAPBBTCA COF was prepared by condensation of 1,3,5-tris-(4aminophenyl)benzene (TAPB) and 1,3,5 benzene-tricarbaldehyde (BTCA) at $\mathrm{rt} 30^{\circ} \mathrm{C}$, according to synthetic methods reported previously by our group (Supporting Information, Section S2). TAPB-TFP COF was prepared by replacing BTCA with 2,4,6trihydroxybenzene-1,3,5-tricarbaldehyde (TFP) during the synthesis (see the Supporting Information, Section S2 for details). The formation of the COFs was confirmed by powder X-ray diffraction (PXRD) and attenuated total reflection Fourier-transform infrared (ATR-FTIR) spectroscopy (Figure 2). Interestingly, although crystalline materials are obtained after $72 \mathrm{~h}$, the appearance of a disordered COF-like product at short reaction times $(2 \mathrm{~h})$ was identified, suggesting the significant presence of local defects within the frameworks.

The gel nature of these 2D COF systems before solvent exchange, inspired us to explore chemical modifications such as metalations directly as gels, conditions under which diffusion and subsequent metal binding to the coordinating groups is favored. [The incorporation of metal sites into COF structures, being an excellent approach to achieve selectivity in heterogeneous catalysis, is commonly carried out post-synthetically on the dried pristine material. Hence, two different one-pot methods to load palladium sites into nitrogen-donor groups within the COFs were studied: First, in situ metalation, where the palladium precursor was directly added to the reacting disordered COF polymer (that is, $2 \mathrm{~h}$ ) and let them further react until the material becomes
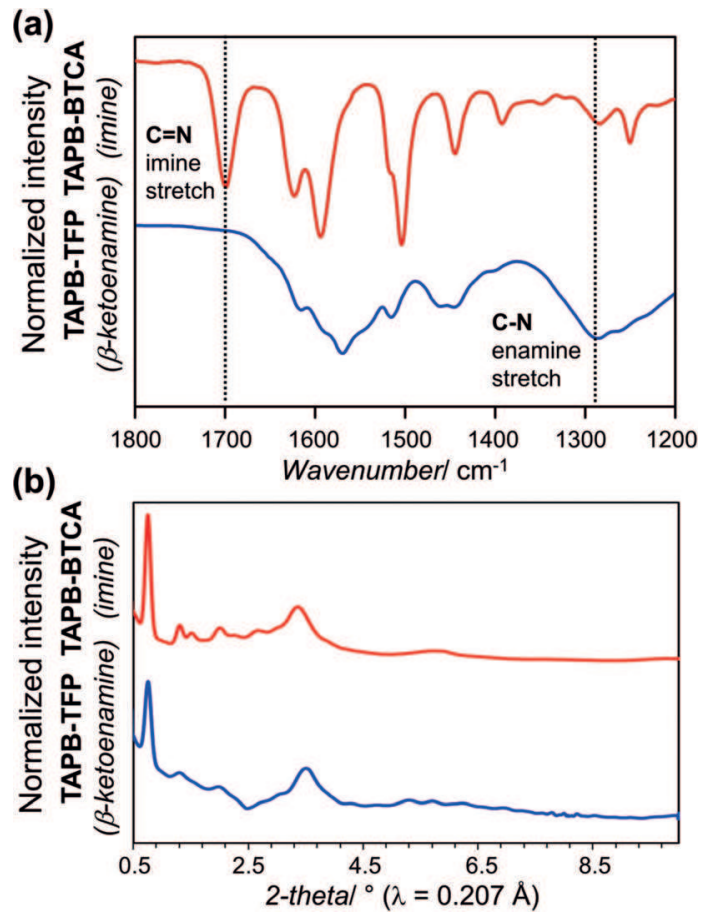

Figure 2. a) FTIR and b) synchrotron PXRD data collected for TAPBBTCA and TAPB-TFP 2D-COF materials. 
crystalline $(72 \mathrm{~h})$. Second, gel metalation, where the palladium precursor is added to the crystalline $\mathrm{COF}$ as a gel and let them react further $(24 \mathrm{~h})$. We hypothesize that palladium metalation achieved through the in situ method would allow the incorporation of palladium into local defects, affording a very interesting defective-system for exploring catalysis.

Chemical composition analyses performed on the COF materials after metalation showed that palladium modification is more efficient following the in situ metalation method, resulting in 14.9 and $11.8 \mathrm{wt} \% \mathrm{Pd}$ loadings for imine and $\beta$ ketoenamine systems, respectively (Supporting Information, Section S4). While targeting same palladium loadings, the gelmetalation afforded materials with systematically lower content (ca. $10 \mathrm{wt} \%$ ) of palladium than those synthesized using the in situ metalation procedure. These results demonstrated that coupling palladium metalation with the dynamic hydrolysis of the imine-linkages, as it occurs during the in situ method, would enhance the efficiency of the metalation step during the synthesis.

Synchrotron powder X-ray diffraction (PXRD) data collected on the palladium-metalated materials showed that crystallinity is retained for both $\mathrm{COF}$ chemistries studied. For the imine-linked TAPB-BTCA materials, diffraction data demonstrates that long-range order is not altered after palladium incorporation within the structure. Interestingly, the palladium-loaded $\beta$-ketoenamine TAPB-TFP system prepared using the in situ method showed improved crystallinity after metalation compared to the pristine material (Figure 3). This observation could indicate that palladium(II) centers may play a defect-healing role during the COF crystallization owing to their Lewis acidity, favoring the stabilization of the $\beta$-ketoenamine over the enol-imine form (that is, favoring the palladium binding to amine groups). On the contrary, this phenomenon is not critical during the metalation of the imine-linked TAPB-BTCA due to the use of acetic acid as catalytic solvent (Supporting Information, Section S2). Pawley analyses of the diffraction data indicated that cell parameters and symmetry remained unaltered after metalation with palladium for all the materials (Supporting Information, Section S3).

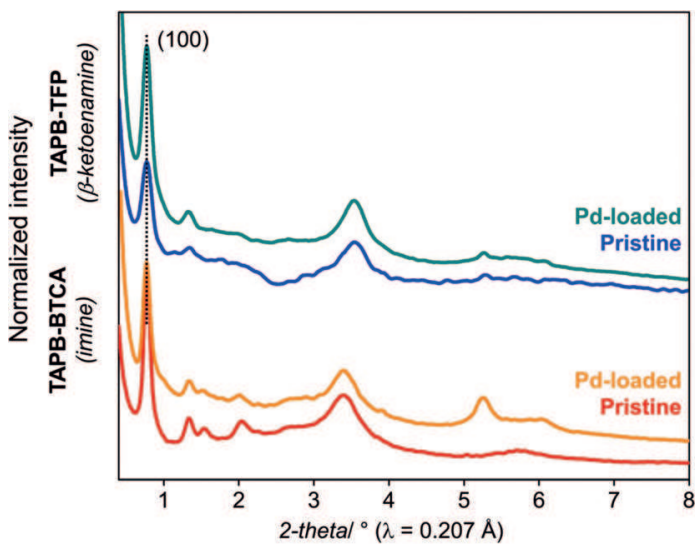

Figure 3. Synchrotron PXRD data collected on the imine TAPB-BTCA and the $\beta$-ketoenamine TAPB-TFP materials after in situ palladium metalation in comparison with the pristine systems.
Nitrogen adsorption-desorption isotherms collected on the activated imine TAPB-BTCA after palladium metalation indicated that porosity is retained. For the imine-linked COF material metalated using the in situ method, a decreased BET surface area value of $494 \mathrm{~m}^{2} \mathrm{~g}^{-1}$ was determined, in comparison with a value of $888 \mathrm{~m}^{2} \mathrm{~g}^{-1}$ determined for the pristine material (Figure 4a; Supporting Information, Table S8.1). Pore size distribution analyses performed using a NLDFT model, indicated a main pore diameter of $15.2 \AA$ for both systems (Figure 4a), in agreement with the framework structure. Additional minor contributions at $12.7 \AA$ and $11.3 \AA$ corresponding to the presence of partially eclipsed layers were also observed, being the population of those less abundant for the palladium-loaded material.

Interestingly, surface area slightly increases after palladium metalation for the $\beta$-ketoenamine BTCA-TFP material. BET values of $664 \mathrm{~m}^{2} \mathrm{~g}^{-1}$ and $550 \mathrm{~m}^{2} \mathrm{~g}^{-1}$ were obtained for the in situ metalated and pristine systems, respectively (Figure 4 b; Supporting Information, Table S8.1). Similar surface areas are obtained for the materials metalated as gels (Supporting Information, Table S8.1). Additionally, pore size distribution of the in situ metalated BTCA-TFP is in good agreement with the values expected from crystallographic data (Figure $4 \mathrm{~b}$ ). These results further corroborated that the palladium centers would favor the occurrence of structural rearrangements coupled to the simultaneous crystallization and tautomeric formation of the $\beta$-ketoenamine form of BTCA-TFP.

To assess the accessibility of the added palladium centers within these two archetypical 2D-COF systems, the catalytic activity of the metalated COF materials was explored for a Suzuki-Miyaura cross-coupling as a model reaction. The coupling of $p$-bromonitrobenzene and phenyl boronic acid using THF as a solvent and base was tested under batch
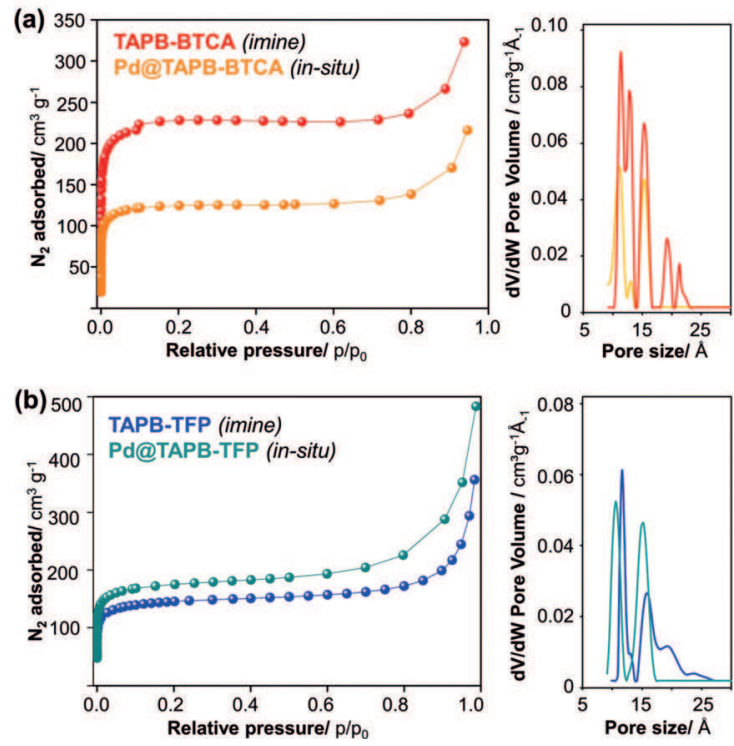

Figure 4. Nitrogen adsorption-desorption isotherms and pore size distribution plots for a) TAPB-BTCA (imine) and b) TAPB-TFP ( $\beta$ ketoenamine) materials after in situ palladium metalation in comparison with the pristine systems. 
conditions at $80^{\circ} \mathrm{C}$ (Supporting Information, Section S12). Under these catalytic conditions, yields of about $70 \%$ were determined after $2 \mathrm{~h}$ reaction time for both TAPB-BTCA and TAPB-TFP systems metalated using the in situ method. On the contrary, lower yields were obtained for both materials metalated using the gel method (Supporting Information, Section S12.1). Additionally, lower yields and higher leaching values were determined for the $\beta$-ketoenamine materials compared to their imine analogues. This suggests a further stabilization of the palladium sites within defective-imine COF systems, in contrast to the defect-free highly stable $\beta$ ketoenamine counterparts.

Being the oxidative addition the most critical step for understanding the reactivity of the palladium centers loaded into the imine-linked TAPB-BTCA in Suzuki-Miyaura couplings, a wider substrate scope was carried out. To assess mechanistic aspects related to this reaction step, different aryl halides containing different stereo-electronic functionalities including electron-withdrawing (Table 1 , entries $\mathbf{1 e}$ and $\mathbf{1 f}$ ) and -donating (Table 1, entries $\mathbf{1 e}, \mathbf{1 g}$ and $\mathbf{1 h}$ ) groups, were selected. In all cases, oxidative addition of the aryl halide to the palladium centers is achieved yielding the desired crosscoupling product (Table 1 ). The results evidence that the catalytic activity of the palladium sites within the TAPBBTCA depends on the electronic properties of the aryl halides, reaching higher yields with electrophilic substrates containing electron withdrawing functionalities in para-positions.

To evaluate the stability of the palladium sites incorporated within these $2 \mathrm{D}-\mathrm{COF}$ materials towards aggregation during the reduction-elimination step, under which palladium(II) is reduced to palladium(0), [5] further characterization was performed. Transmission electron microscopy (TEM) analyses were carried out on the materials before and after catalysis, to identify the formation of palladium nanoparticles (NPs). For both in situ metalated TAPB-BTCA and

Tabelle 1: Suzuki-Miyaura cross-coupling scope using in situ palladiummetalated imine TAPB-BTCA as a catalyst.

Substrate

TAPB-TFP materials, the presence of small palladium NPs of ca. 8 and $11 \mathrm{~nm}$ average size, respectively, was exclusively identified after catalysis, in agreement with the SuzukiMiyaura mechanism (Supporting Information, Section S5). TEM analyses showed that the post-catalysis palladium NPs are homogeneously distributed within these materials. This result evidenced an optimal metalation of the COF structure with palladium following the in situ metalation method (Supporting Information, Section S2). On the contrary, a significant palladium segregation on the external COF surface is determined after catalysis for the gel-metalated COF materials (Supporting Information, Section S5). Diffraction data collected on the post-catalysis materials did not show the presence of bulk palladium black, further supporting the nano-sized nature of the reduced palladium species formed during catalysis.

To probe the local chemical environment of the palladium sites within the $2 \mathrm{D}$-COF systems, Pd $K$-edge extended X-ray absorption fine structure (EXAFS) experiments were performed. Pd $K$-edge EXAFS data collected on representative systems evidenced the occurrence of significant differences in the palladium coordination environment during catalysis, depending on the COF chemistry. For both imine and $\beta$ ketoenamine $\mathrm{COF}$ materials, two contributions centered at about $1.5 \AA$ and about $1.9 \AA$ in the EXAFS spectra (without phase correction) are observed, linked to $\mathrm{Pd}-\mathrm{N}$ and $\mathrm{Pd}-\mathrm{Cl}$ bonds, respectively. Unexpectedly, the signal corresponding to $\mathrm{Pd}-\mathrm{Cl}$ bonds disappears after catalysis, with the concomitance appearance of a contribution centered at about $2.1 \AA$ which is attributed to $\mathrm{Pd}-\mathrm{Br}$ bonds (Figure 5). Importantly, the sharp contribution at about $1.5 \AA$, which is attributed to $\mathrm{Pd}-\mathrm{N}$ bonds remains unaltered after catalysis, demonstrating the actual binding of the palladium species to the COF framework (Figure 5). These results demonstrated that labile chloride ligands are completely exchanged by bromide groups formed during the catalytic cycle in the reaction media, resulting in the main reacting palladium(II) species.

Inspection of $\mathrm{Pd} K$-edge X-ray absorption near-edge structure (XANES) data indicated a subtle shift of the absorption edge determined at $24352 \mathrm{eV}$ to ca. $2 \mathrm{eV}$ lower energy values for all the materials, in agreement with the partial reduction of palladium(II) into palladium(0) during catalysis. EXAFS data obtained on the post-catalysis showed the presence of an expected contribution at about $2.45 \AA$ (without phase correction), which is associated with the formation of $\mathrm{Pd}-\mathrm{Pd}$ bonds after catalysis (Figure 5). This result is in agreement with the formation of $\mathrm{Pd}^{0} \mathrm{NPs}$ during catalysis, as further corroborated by TEM (Supporting Information, Section S5). Importantly, the relative intensity of the $\mathrm{Pd}-\mathrm{Pd}$ bonds contribution with respect to that of the $\mathrm{Pd}-\mathrm{Br}$ bonds is significantly higher for the imine TAPBBTCA materials compared to the $\beta$-ketoenamine counterparts. This evidence agrees with the better catalytic activity obtained for the metalated imine COFs in comparison with their $\beta$-ketoenamine analogues. Furthermore, for the imine TAPB-BTCA metalated through the gel method, the relative intensity of the $\mathrm{Pd}-\mathrm{Pd}$ contributions is clearly larger than the contribution linked to $\mathrm{Pd}-\mathrm{Br}$ bonds, suggesting a larger formation of $\mathrm{Pd}^{0}$ NPs compared to the in situ metalation 

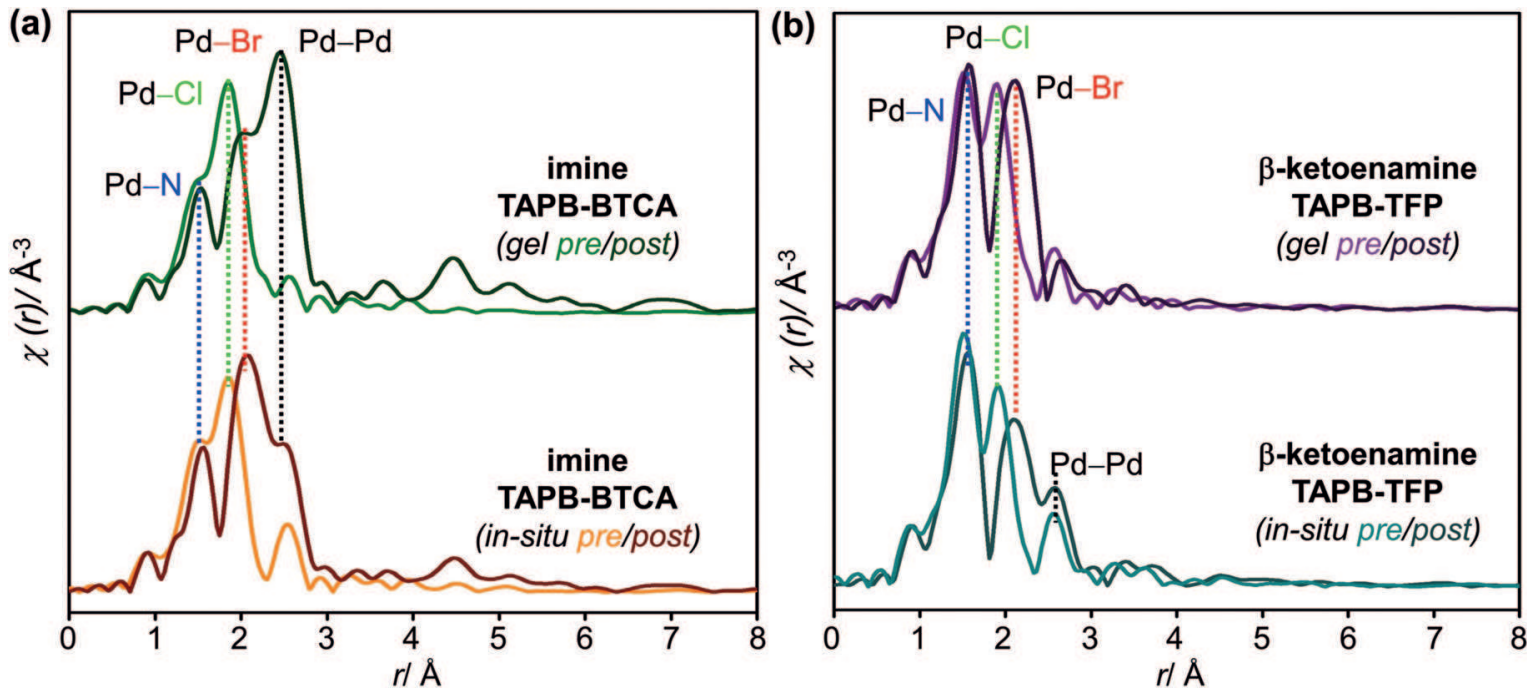

Figure 5. The $k^{3}$-weighted $\chi(r)$ Pd $K$-edge EXAFS spectra of a) metalated imine TAPB-BTCA COF and b) $\beta$-ketoenamine TAPB-TFP COF systems prepared following either the in situ or the gel methods, before and after catalysis.

(Figure 5a). On the contrary, for the less-active material (that is, $\beta$-ketoenamine TAPB-TFP metalated as gel), no significant contributions associated to $\mathrm{Pd}-\mathrm{Pd}$ bonds were identified (Figure $5 \mathrm{~b}$ ). These results demonstrated that the chemical coordination environment of the palladium sites loaded within 2D-COF materials is a structural fingerprint to understand their ultimate catalytic activity.

To better understand the local structural nature of the palladium species loaded through the in situ metalation method, the energetics of defective and pristine imine TAPB-BTCA materials were first explored computationally. We hypothesized that under these synthetic conditions, in which the imine-bonding is highly reversible, the presence of local defects resulting from the hydrolysis of imine linkages to form amine and aldehyde groups might be significant. Structural models showing different relative orientation of the amine and aldehyde groups were computed. Interestingly, periodic calculations demonstrated that imine hydrolysis to give local defects in which the amine and the aldehyde are pointing out towards different directions (trans-defect conformers) are energetically favored by $-5.4 \mathrm{kcal} \mathrm{mol}^{-1}$ (Figure 6; Supporting Information, Figure S11.1). On the contrary, imine hydrolysis to form local defects where amine and aldehydes are pointing towards the same direction (cis-defect conformers) are less favored by $3.4 \mathrm{kcal} \mathrm{mol}^{-1}$, with a formation energy of $-2.0 \mathrm{kcal} \mathrm{mol}^{-1}$ (Figure 6; Supporting Information, Figure S11.1). Interestingly, calculations showed that the energetics of imine hydrolysis within these 2D-COF materials depends on the relative location of the resulting amine and aldehydes groups in the COF pores; more stable defective-COF structures are those for which both aldehyde and amine groups are pointing out towards different directions (trans-conformers, Figure 6). While having related chemistries and similar average structures, these computational studies also demonstrated that defective-TAPB-BTCA structures are stabilized through an energetically more favorable interlayer stacking compared to the idealized defect-free systems (Supporting Information, Tables S11.3 and S11.4).

In a next step, the energetics of the palladium metalation was computationally characterized in detail. Periodic structural models of different palladium-metalated imine-linked TAPB-BTCA systems were computed to explore the binding nature of palladium to different sites for both defect-free and defective-materials. For the defect-free TAPB-BTCA material, DFT calculations showed that palladium(II) only binds to imine groups $\left(\sigma-N_{\mathrm{sp}^{2}}\right)$ from adjacent COF layers, resulting in square-planar geometries. This is the energetically favored

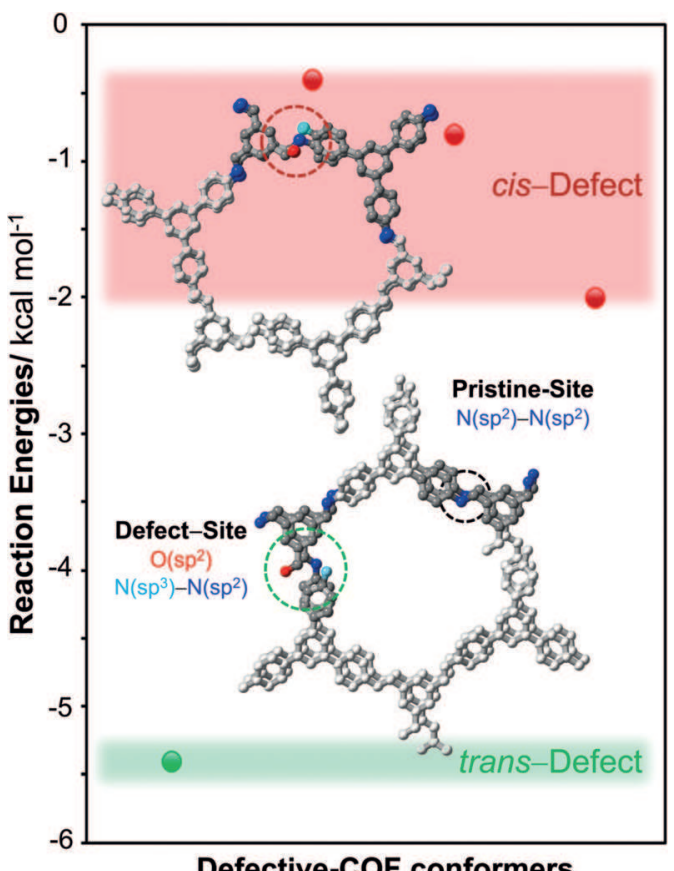

Figure 6. Reaction energies calculated for different defective-COF conformers containing either cis- or trans-defects. 
metalation mechanism with a computed energy gain of $-11.6 \mathrm{kcal} \mathrm{mol}^{-1}$ (Supporting Information, Table S11.3; triclinic, $\alpha \approx 76^{\circ}, \beta \approx 100^{\circ}, \gamma \approx 118^{\circ}$ ). A less favorable conformation with energy gain of $-5.1 \mathrm{kcal} \mathrm{mol}^{-1}$ was also obtained, retaining the native cell symmetry (pseudo-hexagonal, $\alpha=\beta$ $\approx 91^{\circ}, \gamma=120^{\circ}$ ). This result indicated that palladium metalation on the TAPB-BTCA material involves a structural rearrangement, resulting in a more efficient packing of layers. While in both conformations the palladium chemical environment is almost identical, these two models mainly differ in their $\pi-\pi$ stacking and metalation energies. After detailed analysis of the individual bonding interactions for the most stable TAPB-BTCA form (triclinic cell; Supporting Information, Tables S11.3 and S11.4), its corresponding metalation and $\pi-\pi$ stacking energies were estimated to be -23.2 and $-49.3 \mathrm{kcal} \mathrm{mol}^{-1}$, respectively. Interestingly, for the defectfree non-metalated COF, the $\pi-\pi$ energy gain is estimated to be $-48.7 \mathrm{kcal} \mathrm{mol}^{-1}$. These results demonstrated that, after palladium metalation, a more efficient $\pi-\pi$ packing of the $\mathrm{COF}$ layers is identified, as a result of strong interactions with the $\left[\mathrm{PdCl}_{2}\right]$ moieties located within adjacent COF layers.

Interestingly, a much richer structural variety was observed for the palladium metalation on the defective TAPBBTCA system. The DFT calculations suggested that palladium binding is more energetically favored to simultaneously amine $\left(\sigma-N_{\mathrm{sp}^{3}}\right)$ and imine $\left(\sigma-N_{\mathrm{sp}^{2}}\right)$ groups from adjacent layers than to exclusively imine groups, as observed for the defect-free analogue. The difference in relative energy between these two coordination scenarios is low with values of about $3.0 \mathrm{kcalmol}^{-1}$, as anticipated due to the similar chemistries, suggesting the plausible co-existence of both coordination environments. Additionally, these calculations demonstrated that palladium binding to the carbonyl terminations within the local defects is not energetically favored (Table 2). Importantly, palladium-metalation on the amineimine defect site is more energetically favored than on the imine-imine sites within defective COF materials. For low concentration of defects (Figure 7; Supporting Information, Table S11.2), palladium binding to either amine-imine or imine-imine sites has similar energies, suggesting that both coordination environments might coexist. However, with increasing number of defects within the COF, the amineimine site is energetically preferred. This finding suggests that both the formation of local defects (imine hydrolysis) and palladium binding (metalation) to the in situ generated amine

Tabelle 2: Formation energy $\left(\Delta E_{\text {form }}\right)$ linked to the palladium metalation on the imine TAPB-BTCA materials.

\begin{tabular}{|c|c|c|c|}
\hline $\begin{array}{l}\text { Palladium } \\
\text { binding }\end{array}$ & $\begin{array}{l}E_{\mathrm{rel}} \\
{\left[\mathrm{kcal} \mathrm{mol}^{-1}\right]}\end{array}$ & $\begin{array}{l}E_{\mathrm{rel}}{ }^{[\mathrm{a}]} \\
{\left[\mathrm{kcal} \mathrm{mol}^{-1}\right]}\end{array}$ & $\begin{array}{l}E_{\mathrm{rel}}^{[\mathrm{b}]} \\
{\left[\mathrm{kcal} \mathrm{mol}^{-1}\right]}\end{array}$ \\
\hline $\begin{array}{l}\sigma\left(\text { amine }-N_{\mathrm{sp}^{3}}\right) \\
\sigma\left(\text { imine }-N_{\mathrm{sp}^{2}}\right)\end{array}$ & 0 & -9.3 & -3.9 \\
\hline $\begin{array}{l}\sigma\left(\text { imine }-N_{\mathrm{sp}^{2}}\right) \\
\sigma\left(\text { imine }-N_{\mathrm{sp}^{2}}\right)\end{array}$ & +3.0 & -6.3 & -0.9 \\
\hline $\begin{array}{l}\sigma\left(\text { imine }-N_{\mathrm{sp}^{2}}\right) \\
\pi(\mathrm{C}=\mathrm{C})\end{array}$ & +13.2 & +3.9 & +9.3 \\
\hline $\begin{array}{l}\sigma\left(\text { carbonyl }-\mathrm{O}_{\mathrm{sp}^{2}}\right) \\
\pi(\mathrm{N}=\mathrm{C})\end{array}$ & +29.7 & +20.4 & +21.2 \\
\hline
\end{tabular}

[a] Starting from defect-free COF. [b] Starting from defective COF.

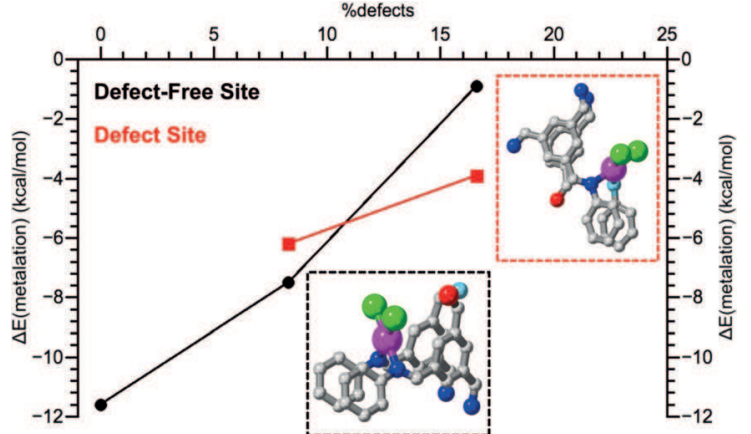

Figure 7. Metalation energy ( $\mathrm{kcal} \mathrm{mol}^{-1}$ ) versus the defect concentration in COF. The \%defect is defined as the number of defect sites per total number of imine groups in the cell. The metalation energies are calculated from: $\mathrm{COF}+\mathrm{PdCl}{ }_{2}\left(\mathrm{CH}_{3} \mathrm{CN}\right)_{2} \rightarrow \mathrm{COF}-\mathrm{PdCl}_{2}+2 \mathrm{CH}_{3} \mathrm{CN}$.

groups might concur. This remarkable result from calculations demonstrated that the defect-sites within TAPB-BTCA are more reactive towards palladium metalation than the imine sites, resulting in different palladium environments than expected together with a more efficient metal loading (Supporting Information, Table S4.1).

To further probe the local structure of the palladium sites beyond the first coordination shell, synchrotron PDF analyses were performed. The PDF technique provides local structural insights to a longer $r$-range than EXAFS, resulting in a unique technique to assess the structural characterization of chemically modified COF systems with compromised periodicity. Differential analysis of the PDF data highlights new and modified atom-atom distances following palladium metalation; by subtracting the PDF of the bare COF to that of the metalated material, new atom-atom distances associated with the added-palladium species are isolated. The differential PDFs (d-PDFs) obtained by subtraction of the PDF collected on bare TAPB-BTCA from that of the corresponding metalated materials before catalysis showed contributions at about $1.9 \AA$ and about $2.3 \AA$ linked to $\mathrm{Pd}-\mathrm{N}$ and $\mathrm{Pd}-\mathrm{Cl}$ distances (Figure $8 \mathrm{a}$ ), respectively, attributed to the coordination environment of the palladium(II) sites. Interestingly, for the material prepared using the in situ method, the d-PDF shows additional sharp contributions up to about $5 \AA$ corresponding to up three shells, in agreement with the periodic DFT model. These results evidenced that the in situ metalation results in well-defined palladium species attached to the defective-COF compared to the gel-method.

After catalysis under conditions relevant for SuzukiMiyaura Coupling, new d-PDF peaks appeared at about $2.7 \AA$ and at longer distances corresponding to $\mathrm{Pd}-\mathrm{Pd}$ correlations within metallic $\mathrm{Pd}^{0}$ NPs with a face centered cubic (fcc) structure. Refinements using a structural model of fcc $\mathrm{Pd}^{0}$ against the experimental d-PDF data provided an estimated average nanocrystal size, assuming a spherical particle model (Figure 9). Refinements were performed in the range of about 2.7-30 A, beyond the contributions associated with the coordination sphere of the palladium(II) sites (that is, a peak at about $2.42 \AA$ is observed linked to $\mathrm{Pd}^{\mathrm{II}}-\mathrm{Br}$ distances formed after catalysis, in agreement with EXAFS data). Average $\mathrm{Pd}^{0}$ crystal dimensions of about 3-4 nm were 

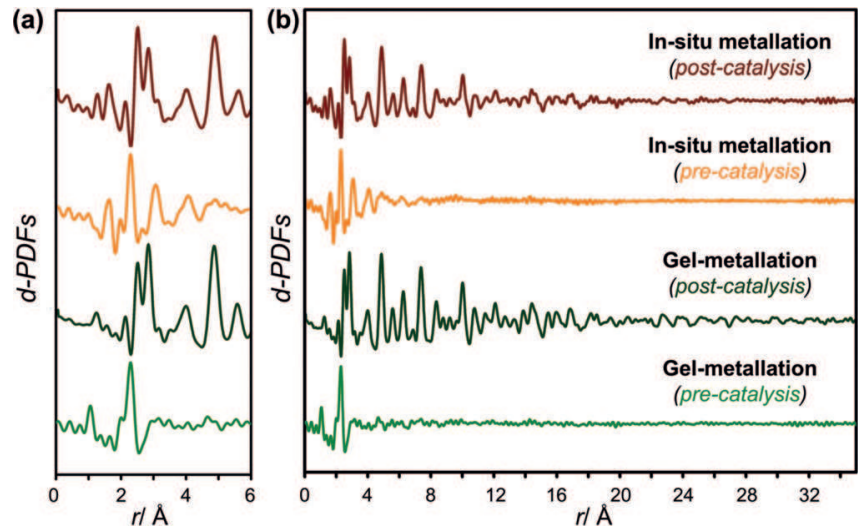

Figure 8. Differential PDFs, d-PDFs, obtained by subtraction of the PDF obtained for pristine TAPB-BTCA COF, showing the contributions attributed to the palladium species before and after catalysis for the different metalation methods used.

estimated for the metalated TAPB-BTCA materials. These results are in agreement with the particle size distribution determined by TEM (ca. 4-15 nm), proving that the palladium nanoparticles are composed of about $1-3 \mathrm{Pd}^{0}$ nanocrystals. These results demonstrated the nano-size nature of the $\mathrm{Pd}^{0}$ crystals formed during the catalytic cycle, being stabilized either on the TAPB-BTCA pores (in situ) or at the external surface (gel; Supporting Information, Section S5).

To truly understand the structural nature of the molecular palladium(II) bromide species formed during SuzukiMiyaura Coupling reactions, combined d-PDF and theoretical DFT studies were further carried out. The formation of bromide anions during catalysis might occur during the oxidative addition step, resulting in the formation of postulated organanopalladium(II) species with characteristic $\mathrm{Pd}-$ $\mathrm{Br}$ bonds. Interestingly, the PDF data collected after catalysis on in situ metalated TAPB-BTCA demonstrated the presence of both palladium(0) nano-crystals of about $3 \mathrm{~nm}$ size

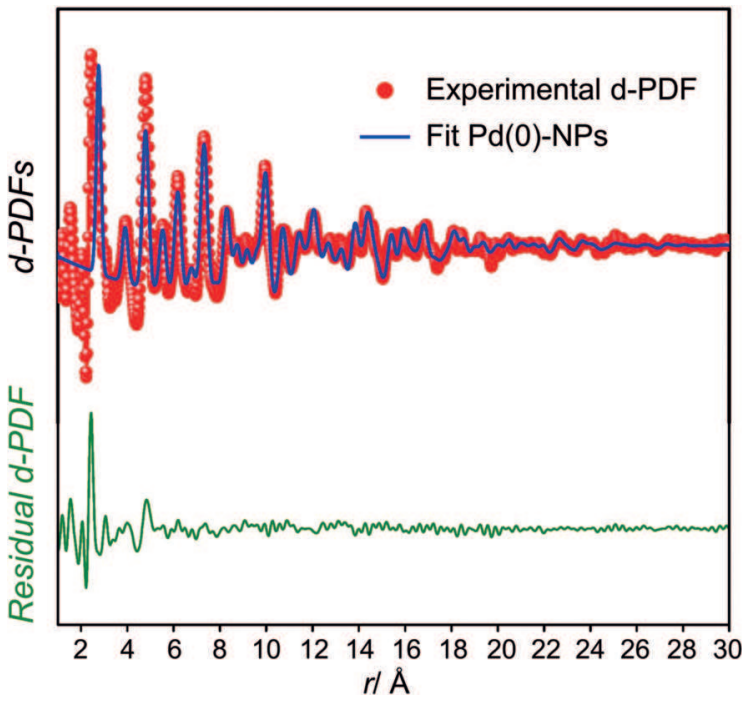

Figure 9. Fit of $\mathrm{fcc} \mathrm{Pd}^{0} \mathrm{NPs}$ to the d-PDF obtained by subtracting the total PDF of in situ metalated TAPB-BTCA from that obtained after catalysis. Features in the residual d-PDF (green) reflect the presence of a well-defined local structure with a main signal attributed to $\mathrm{Pd}$ "- $\mathrm{Br}$ bonds. and well-defined $\mathrm{Pd}^{\mathrm{II}} \mathrm{Br}_{2}$ sites. These palladium(II) species have a well-defined local structure with a main peak centered at about $2.42 \AA$ attributed to $\mathrm{Pd}^{\mathrm{II}}-\mathrm{Br}$ bonds. To explore the energetics of these new formed $\mathrm{Pd}^{\mathrm{II}} \mathrm{Br}_{2}$ sites within the COF pores compared the native dichloride species seen before catalysis, periodic DFT calculations were performed. The residual d-PDF obtained from subtracting the contribution of fcc $\mathrm{Pd}^{0}$ NPs to the d-PDF of in situ metalated TAPB-BTCA after catalysis (Figure 10), highlighted the atomic contributions linked to the new-formed species containing $\mathrm{Pd}^{\mathrm{II}}-\mathrm{Br}$ bonds. Simulated PDF data using the periodic DFT structural model (Figure 10b) matched well with the contributions experimentally observed, demonstrating the single-site local structure of the $\mathrm{Pd}^{\mathrm{II}} \mathrm{Br}_{2}$ reacting sites attached to the COF.

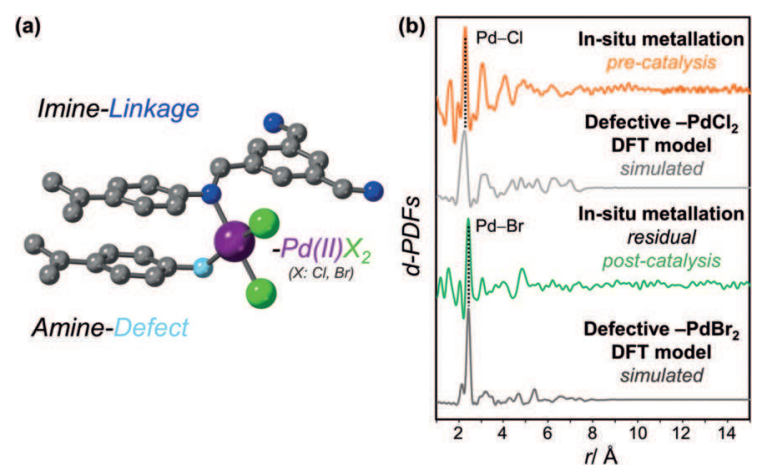

Figure 10. $d$-PDF profiles for in situ metalated TAPB-BTCA COF before and after catalysis, in comparison with the data simulated from the periodic DFT models.

\section{Conclusion}

We have reported the palladation of an imine-linked layered COF (TAPB-BTCA) and its $\beta$-ketoenamine analogue (TAPB-TFP) using a single-step in situ method under which metal binding and COF crystallization occur simultaneously. Advanced synchrotron characterization combining Pd $K$ edge EXAFS and PDF analyses corroborated the incorporation of well defined $\mathrm{Pd}^{\mathrm{II}} \mathrm{Cl}_{2}$ moieties covalently attached to the COF pores. Interestingly, computational studies showed that local defect-sites within TAPB-BTCA, obtained as a result of imine-linkage hydrolysis, are more reactive towards palladation than the ideal pristine sites, resulting in different palladium environments than originally envisaged. We also demonstrated that metalation results in a more energetically favored three-dimensional arrangement, giving a more efficient packing of the COF layers without disrupting the average porous structure. After Suzuki-Miyaura coupling catalysis, a detailed analysis combining EXAFS and PDF corroborated that the palladium reactive species within these COFs are palladium $(0)$ nanocrystals of $3 \mathrm{~nm}$ size. Interestingly, $\mathrm{Pd}^{\mathrm{II}} \mathrm{Br}_{2}$ moieties attached to the COF framework with a well-defined local structure were also identified, which may play a key role in the catalytic cycle. Our results suggest that the incorporation of local defects within imine-linked COF materials could be an interesting strategy to stabilize bonded palladium sites for ultimate catalytic applications. 


\section{Acknowledgements}

We thank the Spanish Ministry of Science, Innovation and Universities (RTI2018-096138-A-I00 and RTI2018-101049-BI00) and the Comunidad de Madrid for funding through the TALENTO grants (2017-T1/IND5148, 2017-T1/AMB-5264, and MAT2016-77608-C3-1-P). A.E.P.-P. and F.Z. acknowledge financial support from the Spanish Ministry of Science and Innovation, through the „María de Maeztu“ Programme for Units of Excellence in R\&D (CEX2018-000805-M). The authors acknowledge the computing facilities of CSUC for providing resources that contributed to the research results reported within this paper. This work was supported by a public grant overseen by the French National Research Agency (ANR) as part of the „Investissements d'Avenir“ program (reference: ANR10-EQPX45). We acknowledge SOLEIL ROCK synchrotron radiation beamline (proposal 20180480). The authors would like to thank Diamond Light Source for rapid-access beamtime and to Dr. Dean S. Keeble to perform X-ray total scattering experiments (proposal CY22812). Part of this research were carried out at P02.1 beamline at DESY (proposals I-20170717 EC and I-20190208 EC), a member of the Helmholtz Association (HGF). We would like to thank Dr. Michael Wharmby for assistance during the experiment at P02.1. The research leading to this result has been supported by the project CALIPSOplus under the Grant Agreement 730872 from the EU Framework Programme for Research and Innovation HORIZON 2020,. I.R.-M. acknowledges a FPI-UAM 2019 fellowship from Universidad Autónoma de Madrid. P.A. acknowledges the financial support from "Ayudas para Contratos Predoctorales para la Formación de Doctores, Program of MINECO (Grant BES-2017-070838).

\section{Conflict of interest}

The authors declare no conflict of interest.

Stichwörter: cross-coupling catalysis - DFT modeling layered covalent organic frameworks - pair distribution function

[1] O. M. Yaghi, . Am. Chem. Soc. 2016, 138, 15507-15509.

[2] O. M. Yaghi, W. J. Waller, F. Gándara, Acc. Chem. Res. 2015, 48, $3053-3063$.

[3] W. Ji, L. Xiao, Y. Ling, C. Ching, M. Matsumoto, R. P. Bisbey, D. E. Helbling, W. R. Dichtel, T. Am. Chem. Soc. 2018, 140. 12677-12681.

[4] P. Albacete, A. López-Moreno, S. Mena-Hernando, A. E. Platero-Prats, E. M. Pérez, F. Zamora, Chem. Commun. 2019, $55,1382-1385$.

[5] Q. Gao, X. Li, G. H. Ning, K. Leng, B. Tian, C. Liu, W. Tang, H. Sen Xu, K. P. Loh, Chem. Commun. 2018, 54, 2349-2352

[6] C. R. Mulzer, L. Shen, R. P. Bisbey, J. R. McKone, N. Zhang, H. D. Abruña, W. R. Dichtel, ACS Cent. Sci. 2016, 2, 667-673

[7] A. Halder, M. Ghosh, A. Khayum, S. Bera, M. Addicoat, Himadri, S. Sasmal, S. Karak, S. Kurungot, R. Banerjee, T. Am. Chem. Soc. 2018, 140, 10941-10945,

[8] C. R. Deblase, K. E. Silberstein, T. T. Truong, H. D. Abruña, W. R. Dichtel, T. Am. Chem. Soc. 2013, 135, 16821-16824.
[9] P. Albacete, J. I. Martínez, X. Li, A. López-Moreno, S. MenaHernando, A. E. Platero-Prats, C. Montoro, K. P. Loh, E. M. Pérez, F. Zamora, T. Am. Chem. Soc. 2018, 140, 12922-12929.

[10] M. Bhadra, S. Kandambeth, M. K. Sahoo, M. Addicoat, E. Balaraman, R. Banerjee, . Am. Chem. Soc. 2019, 141, 61526156.

[11] H. B. Aiyappa, J. Thote, D. B. Shinde, R. Banerjee, S. Kurungot, Chem. Mater. 2016, 28, 4375-4379.

[12] F. J. Uribe-Romo, J. R. Hunt, H. Furukawa, C. Klöck, M. O'Keeffe, O. M. Yaghi, . Am. Chem. Soc. 2009, 131, 4570-4571.

[13] E. Vitaku, W. R. Dichtel, . Am. Chem. Soc. 2017, 139, 12911 12914

[14] S. Kandambeth, K. Dey, R. Banerjee, t. Am. Chem. Soc. 2019 141,1807-1822.

[15] F. Segura, L. Mancheño, M. J. Zamora, Chem. Soc. Rev. 2016, 45, $5635-5671$

[16] A. Halder, S. Karak, M. Addicoat, S. Bera, A. Chakraborty, S. H. Kunjattu, P. Pachfule, T. Heine, R. Banerjee, Angew. Chem. Int. Ed. 2018, 57, 5797-5802; Angew. Chem. 2018, 130, 5899-5904.

[17] S. Kandambeth, A. Mallick, B. Lukose, M. V. Mane, T. Heine, R. Banerjee, . Am. Chem. Soc. 2012, 134, 19524-19527.

[18] G. Das, D. Balaji Shinde, S. Kandambeth, B. P. Biswal, R. Banerjee, Chem. Commun. 2014, 50, 12615-12618.

[19] M. C. Daugherty, E. Vitaku, R. L. Li, A. M. Evans, A. D. Chavez, W. R. Dichtel, Chem. Commun. 2019, 55, 2680-2683,

[20] P. Pachfule, S. Kandambeth, D. Díaz Díaz, R. Banerjee, Chem. Commun. 2014, 50,3169-3172.

[21] D. Rodríguez-San-Miguel, A. Yazdi, V. Guillerm, J. PérezCarvajal, V. Puntes, D. Maspoch, F. Zamora, Chem. Eur. J. 2017, $23,8623-8627$

[22] H. C. Ma, J. L. Kan, G. J. Chen, C. X. Chen, Y. Bin Dong, Chem. Mater. 2017,29,6518-6524.

[23] M. Bhadra, H. S. Sasmal, A. Basu, S. P. Midya, S. Kandambeth, P. Pachfule, E. Balaraman, R. Banerjee, ACS Appl. Mater. Interfaces 2017, 9, 13785-13793

[24] C. C. C. Johansson Seechurn, M. O. Kitching, T. J. Colacot, V. Snieckus, Angew. Chem. Int. Ed. 2012, 51, 5062-5085; Angew. Chem. 2012, 124, 5150-5174

[25] S. Y. Ding, J. Gao, Q. Wang, Y. Zhang, W. G. Song, C. Y. Su, W. Wang, T. Am. Chem. Soc. 2011, 133, 19816-19822.

[26] P. Pachfule, M. K. Panda, S. Kandambeth, S. M. Shivaprasad, D. D. Díaz, R. Banerjee, . Mater. Chem. A 2014, 2, 7944-7952

[27] D. Mullangi, S. Nandi, S. Shalini, S. Sreedhala, C. P. Vinod, R. Vaidhyanathan, Sci. Rep. 2015, 5, 10876.

[28] S. Lin, Y. Hou, X. Deng, H. Wang, S. Sun, X. Zhang, RSC Adv. 2015, 5, 41017-41024.

[29] R. S. B. Gonçalves, A. B. V. Deoliveira, H. C. Sindra, B. S. Archanjo, M. E. Mendoza, L. S. A. Carneiro, C. D. Buarque, P. M. Esteves, ChemCatChem 2016, 8, 743-750.

[30] A. de la Peña Ruigómez, D. Rodríguez-San-Miguel, K. C. Stylianou, M. Cavallini, D. Gentili, F. Liscio, S. Milita, O. M. Roscioni, M. L. Ruiz-González, C. Carbonell, et al., Chem. Eur. T. 2015,21, 10666-10670.

[31] J. Romero, D. Rodriguez-San-Miguel, A. Ribera, R. MasBallesté, T. F. Otero, I. Manet, F. Licio, G. Abellán, F. Zamora, E. Coronado, T. Mater. Chem. A 2017, 5, 4343-4351.

[32] N. Yuan, V. Pascanu, Z. Huang, A. Valiente, N. Heidenreich, S. Leubner, A. K. Inge, J. Gaar, N. Stock, I. Persson, et al., T. Am. Chem. Soc. 2018, 140, 8206-8217

Manuskript erhalten: 22. März 2020

Veränderte Fassung erhalten: 21. April 2020

Akzeptierte Fassung online: 25. April 2020

Endgültige Fassung online: 28. Mai 2020 